\title{
THz Waveguide and Bends based on Metallic Photonic Crystals
}

\author{
E. Degirmenci, Student Member, IEEE, F. Surre, Member, IEEE, \\ P. Landais, Member, IEEE \\ Research Institute for Networks and Communications Engineering (RINCE) \\ School of Electronics Engineering, Dublin City University, Dublin 9, Ireland
}

\begin{abstract}
Metallic band-gap structures for $\mathrm{THz}$ waveguide and bends have been simulated using the finite element method. Transmission characteristics have been obtained for various square array waveguide designs.
\end{abstract}

$\mathrm{T}$ ERAHERTZ (THz) waves refer to electromagnetic (EM) radiation in a frequency band between $0.3 \mathrm{THz}$ and 10 $\mathrm{THz}$, corresponding to wavelengths in the sub-millimeter range. THz science and technology have been developing very rapidly for their potential applications in variety of areas, such as imaging, security, spectroscopy and communication. With the rise of $\mathrm{THz}$ frequency based technology and new developments in generating $\mathrm{THz}$ radiation, guiding $\mathrm{THz}$ waves in an efficient way with low-loss, high performance become of a key relevance. Starting from early studies on $\mathrm{THz}$, new solutions as well as the old ones from microwave or optical frequencies have been proposed for guiding. Among the new solution we can mention metal wires [1], sapphire fiber [2], plastic ribbon waveguide [3], parallel-plate waveguide [4], and photonic crystals $[5,6]$. Each of them presents their own individual challenges and advantages.

The purpose of this study is to design a waveguide to guide and manipulate the $\mathrm{THz}$ radiation in an efficient way in order to increase the performance of the actual $\mathrm{THz}$ devices. For this aim we propose metallic photonic band-gap (PBG) crystal waveguides as an efficient solution. Photonic crystals are periodically distributed materials allowing the control of electromagnetic waves propagation within the crystals. Under condition of refractive index difference between medium and photonic crystal material, the propagation of an EM wave is forbidden within a frequency range called the photonic band gap. These photonic crystal structures can be used to confine or to propagate EM waves within defects introduced in their structure; otherwise EM waves are prevented to propagate inside the crystal structure. For interconnection to other devices, waveguides are required to have flexibility of bending. However, bends introduce losses arising from total internal reflection. To overcome this problem, photonic crystals have been studied with the advantages of low losses and low dispersion properties of photonic crystals and almost perfect transmission has been obtained around sharp bends [7]. Nevertheless, metallic band-gap crystals have demonstrated important advantages over the dielectric photonic crystals, such as wider band-gaps and smaller sizes [8]. Metallic photonic crystals have been studied theoretically and experimentally mostly in microwave and optical frequencies. [9-12].

The waveguide presented here photonic crystal waveguide and waveguide bends in which a line defect is introduced in photonic crystal structure by removing one row of rods in the middle of square lattice (Fig.1). Simulation has been carried out using one of the commercially available tools based on the Finite Element Method (FEM) in 2D. Maxwell's equations are solved applying non-reflecting boundary conditions in order to simulate the wave propagation in a waveguide design. Due to the $2 \mathrm{D}$ simulation, this cavity is considered only allowing light transmitted in E-polarization in which the electric field is parallel to the rod axis. The electromagnetic wave confinement is provided using the contrast between metal and vacuum permittivities.
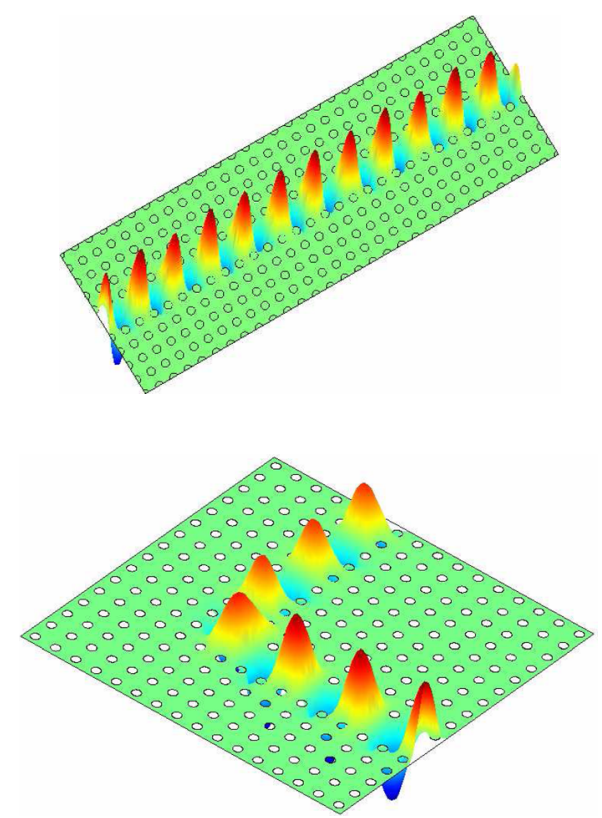

Figure 1 Propagation in a photonic crystal waveguide and waveguide bend. Rods shown in circles are made of copper.

\section{RESULTS}

The two-dimensional metallic photonic crystal is used for 
designing waveguide and bends in square array of copper rods. The dielectric function of copper is simulated by using the frequency-dependent Drude model [13]. Waveguide design parameters: a, the lattice constant is set at $50 \mu \mathrm{m}$ and the rod's radius, $r$ at $0.4 \mathrm{a}$. For radius to lattice constant ratio larger than 0.4 , a band-gap exists for the whole $\mathrm{THz}$ frequency range [14$15]$. In the rest of this paper, the r/a ratio is fixed at 0.4 for our waveguide designs.

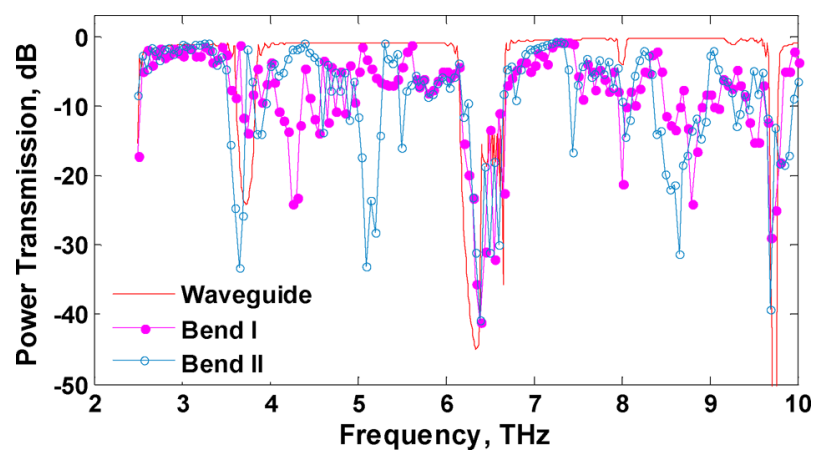

Figure 2 Transmission spectra of a waveguide and 2 bend structures, see insert of Fig. 3 .

Power transmission and reflection characteristics of $\mathrm{THz}$ metallic band-gap crystal waveguide and bend configurations have been shown in Fig.2 and Fig.3, respectively. As it seen in Fig.2, a very good transmission has been obtained at frequencies between 2.7-3.45, 4-6 and 6.85-9.15 THz for square array waveguide. In these frequency bands the transmission achieved is of $97 \%$ of the original injected signal. By comparison the transmission amplitude of $\mathrm{Cu}$ rectangular waveguide of the same length is $7 \%$. Metallic photonic crystals have shown better characteristics than metallic waveguide as the length of waveguide increases. The reduction of the losses in our structure is due to the fact that to guide the $\mathrm{THz}$ wave less metallic material is required. The reason of dips observed in transmission spectrum is due to higher mode propagating above $3.45 \mathrm{THz}$.

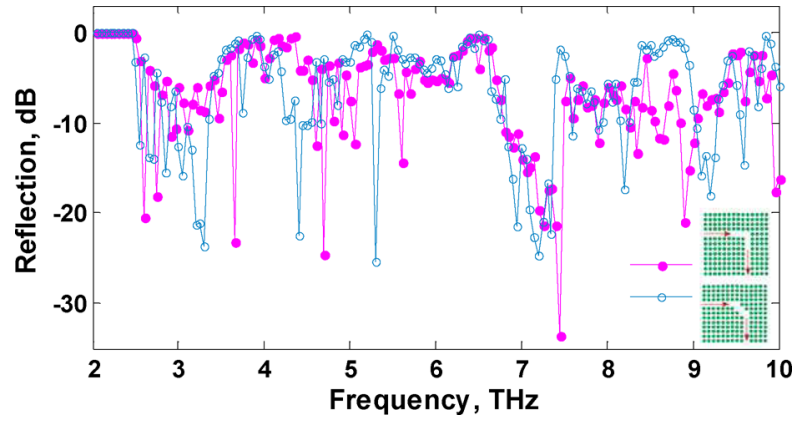

Figure 3 Reflection spectra of waveguide bend structures bend I and bend II The schematic and direction of propagation can be seen on the inset corresponding to the legend of figure.

As it seen from the inset on the Fig.3, two types of bend structure are studied. First one is a $90^{\circ}$ sharp corner (Bend I), the other one is smoother $90^{\circ}$ bend (Bend II). Similar trend can be seen on both bend structures to the metallic photonic crystal waveguide, losses are due to the back reflections on the corners. Back reflections are due to the rods edge of corners, when wave reaches to the corners it will be reflected back. From Fig. 3 the difference of performances of bend structures can be easily seen that sharp bend has less back reflection than the smoother bend.

\section{CONCLusions}

We simulated 2-D metallic photonic crystal waveguide and bend structure based on square lattice array with copper at $\mathrm{THz}$ frequency range. Over a wide frequency ranges consistently high transmission characteristics is obtained in square lattice waveguide, and similar trend also obtained with bends with losses due to the back reflection. Metallic photonic crystals are a good candidate for $\mathrm{THz}$ wave guiding.

\section{ACKNOWLEDGMENTS}

This work is supported by Enterprise Ireland (Project No. 202/06/0286) and the HEA PRTLI4 INSPIRE programme.

\section{REFERENCES}

[1] D.K. Wang and D.M. Mittleman, "Metal Wires for Terahertz Wave Guiding," Nature, vol. 432, pp. 376-379, Sep. 2004.

[2] S.P. Jamison, R.W. Mcgowan, and D. Grischkowsky, "Single-Mode Waveguide Propagation and Reshaping of Sub-Ps Terahertz Pulses in Sapphire Fibers, " App. Phys. Lett., vol. 76, pp. 1987-1989, Apr. 2000.

[3] R. Mendis and D. Grischkowsky, "Plastic Ribbon THz Waveguides," $J$. App. Phys, vol. 88, pp. 7-9, Oct. 2000.

[4] A. Bingham, Y.Zhao and D. Grischkowsky, "THz Parallel Plate Waveguides," App. Phys. Lett., vol. 87, 051105, Jul. 2005.

[5] C. Lin, C. Chen, G.J. Schneider, P. Yao, S. Shi, and D.W. Prather, "Wavelength Scale Terahertz Two-Dimensional Photonic Crystal Waveguides, " Opt Exp., vol. 12, pp. 5723-5728, Nov. 2004

[6] C. Chi, H. Wang, S. Pai, W. Lai, S. Horng, and R.S. Huang, "Fabrication and Characterization of Terahertz Photonic Crystals, "Proceedings of SPIE, vol. 46 pp. 19-30, 2002

[7] A. Mekis, J. C. Chen, I. Kurland, Shanhui Fan, Pierre R. Villeneuve, and J. D. Joannopoulos, "High Transmission through Sharp Bends in Photonic Crystal Waveguides, " Phys. Rev. Lett. Vol. 77, pp. 3787 3790 Jun. 1996

[8] V. Kuzmiak, A. Maradudin, and F. Pincemin, "Photonic Band Structures of Two-Dimensional Systems Containing Metallic Components, "Phys. Rev. B, vol. 50, pp. 16835-16844, Dec. 1994

[9] I. El-Kady, M. M. Sigalas, R. Biswas, K. M. Ho, and C. M. Soukoulis, "Metallic photonic crystals at optical wavelengths," Phys. Rev. B, vol 62, 15299 - 15302, Jun. 2000

[10] M.M Sigalas, C.T, Chan, K.M. Ho and C.M. Soukoulis, "Metallic photonic band-gap materials, " Phys. Rev. B vol. 52, pp.11744-11751, Oct. 1995

[11] F. Gadot, A. de Lustrac, J.M. Lourtioz, "High Transmission Defect Modes in Two-dimensional Metallic Photonic Crystals, " J. Appl. Phys. vol. 85 , pp. $8499-8501$, Jun. 1999

[12] M. Bayindir, E. Cubukcu, I. Bulu, T. Tut, E. Ozbay, and C.M. Soukoulis, "Photonic Band Gaps, Defect Characterisics, and Waveguiding in TwoDimensional Disordered Dielectric and Metallic Photonic Crystals, " Phys. Rev. B vol. 64, 195113, Oct. 2001

[13] M.A. Ordal, L.L. Long, R.J. Bell, S.E. Bell, R.R. Bell, R.W. Alexander, and C.A. Ward, "Optical Properties of The Metals Al, Co, Cu, Au, Fe, Pb, Ni, Pd," App. Opt., vol. 22, pp. 1099-1120, Nov.1983

[14] E.I. Smirnova, C. Chen, M.A. Shapiro, J.R. Sirigiri, R.J. Temkin, "Simulation of Photonic Band Gaps in Metal Rod Lattices For Microwave Applications," J. App. Phys, vol. 91, Feb.2002

[15] Y. Kokubo, T. Kawai, and I. Ohtal, "Photonic Crystal Waveguides with Inner Metal- Coated Posts at Millimeter-Wave Frequency Range," 34th Europian Microwave Conference, pp. 1137-1140, 2004 